\title{
Obesity induced by cafeteria feeding and pregnancy outcome in the rat
}

\author{
Asli Akyol, Simon C. Langley-Evans and Sarah McMullen* \\ School of Biosciences, University of Nottingham, Sutton Bonington, Loughborough LE12 5RD, UK
}

(Received 2 March 2009 - Revised 1 June 2009 - Accepted 5 June 2009 - First published online 22 July 2009)

Obesity during pregnancy has major consequences for maternal and neonatal health, but the long-term effects on the offspring are less clear. It is not known whether the effects observed in animal models are a result of maternal obesity per se or of the high-fat diets used to induce obesity. This investigation aimed to develop a model for the evaluation of the independent effects of cafeteria feeding and maternal obesity, considering their impact on plasma volume expansion, circulating metabolites, and fetal and placental growth. Wistar rats were fed a control or cafeteria diet from weaning. After 8 weeks, all animals were mated and half of the animals within each group were crossed-over to the alternative diet. This generated four treatment groups, differing in their pre-gestational and gestational diets. Half of the animals were culled at day 5 of gestation and the remainder at day 20. Maternal body composition, blood volume and circulating glucose, TAG and cholesterol were determined. Cafeteria feeding was effective in inducing obesity, as demonstrated by increased fat depot weights and total body fat, without impacting upon reproductive success or circulating lipid concentrations. The study successfully demonstrated that there were differential effects of maternal body fatness and diet upon fetal and placental growth, with pre-gestational obesity leading to lower fetal weight at day 20 of gestation $(P<0$-001). The model will provide a useful vehicle for the investigation of the complex interactions between dietary- and obesity-related factors during pregnancy in their effects on fetal development and postnatal metabolic function.

Pregnancy: Obesity: Rats: Cafeteria feeding

Obesity during pregnancy has major consequences for maternal and neonatal health. Rising levels of childhood obesity in Europe ${ }^{(1)}$ and the $\mathrm{USA}^{(2)}$ indicate that the prevalence of obesity in women of reproductive age is set to rise in the future. Indeed, the Foresight Report ${ }^{(3)}$ estimated $13 \%$ of 21- to 30-year-old and $22 \%$ of 31- to 40-year-old females in England to be obese in 2007, and projected that this would rise to 30 and $47 \%$ respectively by 2050 . A huge body of evidence demonstrates the adverse consequences of obesity on pregnancy outcome. Women who are overweight or obese at conception are more likely to develop hypertensive, diabetic or thrombotic complications, and to deliver macrosomic babies ${ }^{(4,5)}$. Associated with these risks are increased rates of delivery complications, postpartum haemorrhage and Caesarean section ${ }^{(4,6,7)}$. The cumulative effect is an increased risk of maternal, fetal and neonatal mortality.

In contrast to these more immediate consequences of maternal obesity, the evidence relating to the consequences of maternal obesity for the long-term health of the offspring is less clear. Human epidemiological studies have found positive associations between maternal BMI in pregnancy and the risk of obesity in the offspring in later life ${ }^{(8-10)}$. However, this may reflect the transmission of genetic or shared familial lifestyle factors across generations. Increased strength of the maternal-offspring association in comparison with the paternal-offspring association suggests that there is an intrauterine component involved ${ }^{(11)}$, but this has not been observed in all studies ${ }^{(12,13)}$. Other studies have focused on high birth weight $^{(14,15)}$ and gestational diabetes ${ }^{(16-18)}$ as markers of fetal overnutrition and demonstrate a consistent, although not necessarily independent, association with increased adiposity and metabolic risk in the offspring.

Studies of animal models have confirmed that both underand overnutrition during pregnancy can promote metabolic risk in later life ${ }^{(19)}$. Such studies have demonstrated that early life factors contribute to the development of insulin resistance $^{(6)}$, hypertension $^{(7,8)}$ and endothelial dysfunction ${ }^{(9)}$. Reflecting the human literature, many of the early animal studies modelling the developmental origins of disease focused on nutrient restriction during pregnancy. However, more recent work has shown that maternal high-fat feeding during pregnancy can have similar postnatal consequences. Offspring from dams fed a maternal obesity-inducing, hyperenergetic diet before and during pregnancy have been shown to exhibit disturbed glucose and lipid homeostasis and greater adiposity in both the mouse ${ }^{(20)}$ and the rat ${ }^{(21)}$. Similarly, Bayol et al. ${ }^{(22,23)}$ showed that cafeteria feeding during pregnancy could predispose to adiposity and altered feeding behaviour in the rat. Collectively, these studies demonstrate that feeding high-fat, obesity-inducing diets during pregnancy in

Abbreviations: CAF, cafeteria diet; CAF-CAF, cafeteria diet pre-gestation and during pregnancy; CAF-CON, cafeteria diet pre-gestation and control chow diet during pregnancy; $\mathrm{CON}$, control chow diet; CON-CAF, control chow diet pre-gestation and cafeteria diet during pregnancy; CON-CON, control chow diet pre-gestation and during pregnancy

* Corresponding author: Dr Sarah McMullen, fax +44 115951 6122, email sarah.mcmullen@Nottingham.ac.uk 
rodent models can lead to permanent alterations in postnatal physiological function, promoting adiposity and CVD risk.

What is not clear from these studies is whether maternal obesity acts as a programming agent per se or whether other aspects of the obesity-inducing diet (for example, lower protein or higher fat content) drive the fetal responses. Shankar et al. ${ }^{(24)}$ addressed this issue by rendering rats obese with a liquid high-fat diet fed by intragastric cannulation, then transferring obese animals to a control diet at mating and cross-fostering pups to lean dams at birth. In comparison with those from lean dams, the offspring of obese dams exhibited increased adiposity and insulin resistance. This study therefore demonstrates an independent effect of maternal obesity, specifically during the fetal period, on postnatal disease risk. The aim of the present investigation was to develop a robust model for the evaluation of the independent effects of a cafeteria diet and the intra-uterine environment associated with maternal obesity. The primary objective of the present study was to evaluate the impact of cafeteria feeding during pre-pregnancy and/or the pregnancy period on maternal body composition and fecundity. Having demonstrated the effectiveness of the feeding regimen in inducing obesity without impacting on reproductive success, the secondary objective was to assess the impact of obesity and cafeteria feeding on maternal adaptation to pregnancy, maternal circulating glucose and lipid status, and fetal and placental growth.

\section{Methods \\ Animal procedures}

The experiments were performed under licence from the Home Office in accordance with the 1986 Animals Act (Scientific Procedure). All animals were housed individually in plastic cages and subjected to a $12 \mathrm{~h}$ light-dark cycle at a temperature of $20-22^{\circ} \mathrm{C}$ and $45 \%$ humidity. The animals were housed on shavings and had ad libitum access to food and water at all times. Virgin female Wistar rats (aged 3 weeks; $n$ 47) were randomly allocated to be fed either a control chow diet alone (CON; $n$ 23) or a control chow diet alongside a random selection of highly energetic and palatable human foods (cafeteria diet; CAF; $n$ 23). These included biscuits, potato crisps, fruit and nut chocolate, Mars bars, cheddar cheese, golden syrup cake, pork pie, cocktail sausages, liver and bacon pâté, strawberry jam and peanuts. Four of the cafeteria foods were provided in a bowl on the cage floor daily in excess quantities. The foods provided were altered daily, to maintain variety, by replacing two of the foods with new items. Hence the animals did not receive the same foods for more than two consecutive days at a time. The chow diet (B\&K Universal Ltd, Hull, UK) and cafeteria diet foods were individually weighed in and out of the cage between 09.00 and 10.00 hours daily. Daily intakes of energy, macronutrients and micronutrients were calculated from the manufacturers' data. Weight loss due to evaporation was measured in triplicate samples of each individual food item placed in empty cages. The average daily percentage change in the weight of foods ranged from 0 to $6.2 \%$ and corresponded to an average overestimation of energy intake by $2.51 \%(7.5 \mathrm{~kJ} / \mathrm{d})$, which can be considered within an acceptable error of measurement. Body weights of the animals were measured between 09.00 and 10.00 hours daily. Diets were introduced from weaning to allow a sufficient period of cafeteria feeding to induce obesity before mating at age 10 weeks.

After 8 weeks of control or cafeteria feeding, all rats were paired with a Wistar stud male and mating confirmed by the appearance of a semen plug. In order to separate the effects of maternal cafeteria feeding from the effects of maternal obesity, half of the animals from the control group were randomly allocated to the cafeteria diet (CON-CAF; $n$ 11) and half of the animals from the cafeteria group were randomly allocated to the control diet (CAF-CON; $n$ 11) upon confirmation of mating. The remaining animals within each group were maintained on their pre-gestational diets (CON-CON; $n 12$ and CAF-CAF; $n$ 12).

Rats were terminally anaesthetised using isoflurane on day 5 or 20 of gestation, for the measurement of plasma volume expansion using the Evans blue procedure ${ }^{(25)}$. Briefly, a cannula was inserted into the left iliac vein and a baseline blood sample removed before administration of $0.3 \mathrm{ml}$ Evans blue dye $(0.5 \mathrm{mg} / \mathrm{ml})$. After $5 \mathrm{~min}$, a second blood sample was removed. Baseline and $5 \mathrm{~min}$ blood samples were centrifuged at $13000 \mathrm{rpm}$ and the absorbance of plasma samples determined at a wavelength of $610 \mathrm{~nm}$. A standard curve was constructed by linear regression analysis of absorbance values obtained from known concentrations of Evans blue dye $(0.001-0.15 \mathrm{mg} / \mathrm{ml})$. Plasma volume was estimated by calculating the dilution of the dye. Animals were euthanased by injection of pentobarbitone and death confirmed by cervical dislocation. Maternal gonadal fad pad, peri-renal fad pad and liver were weighed and a sample stored for further analysis. Fetal body weight, liver, brain, kidneys, heart and placenta were weighed and sampled from the animals that were culled on day 20 of gestation.

\section{Body composition}

Maternal carcass composition was determined in all animals by chemical analysis. Whole carcasses were oven-dried to determine the body water content as previously described ${ }^{(26)}$. The dried carcasses were homogenised and sampled for estimation of $\mathrm{N}$ content by the Kjehldal method and for fat content by Soxhlet extraction.

\section{Plasma metabolites}

Plasma metabolite analyses were performed on baseline samples collected before administration of the Evans blue dye. Total plasma cholesterol and total TAG were assayed using commercially available kits (Thermo Life Sciences, Basingstoke, Hants, UK). Plasma glucose was assayed using an adapted protocol based on the glucose oxidase method $^{(27)}$. A standard curve was constructed by linear regression analysis of absorbance values of known concentrations of glucose $(0-2 \mu \mathrm{g}$ glucose). Plasma samples were diluted 1:5 with phosphate buffer. In duplicate, $10 \mu \mathrm{l}$ of sample and $200 \mu \mathrm{l}$ of glucose reagent were added to the wells of a microtitre plate. The plate was incubated at $37^{\circ} \mathrm{C}$ for $15 \mathrm{~min}$ and read at an absorbance of $620 \mathrm{~nm}$ (Sunrise $^{\mathrm{TM}}$, Tecan Group Ltd, Switzerland). 


\section{Statistical analysis}

All data were analysed using the Statistical Package for Social Sciences (version 16; SPSS, Inc., Chicago, IL, USA). The effect of pre-gestational and gestational diet on maternal and fetal outcomes was assessed using a general linear model ANOVA (fixed factors, maternal diet, sex and age). Where longitudinal data were available (for example, weekly body weights or energy intake), the week of study was used in a repeated-measures analysis. Values are expressed as mean values with their standard errors. $P<0.05$ was considered statistically significant. No post hoc analyses were performed.

\section{Results}

\section{Nutrient intakes}

When offered the cafeteria diet, weanling rats markedly increased their food and hence energy intake. There was some adjustment to energy intake after the first $7-10 \mathrm{~d}$ of cafeteria feeding, but average intakes over the pre-pregnancy period remained significantly higher in cafeteria-fed animals when compared with chow-fed animals (Fig. 1, Table 1; $P<0.001)$. Cafeteria-fed animals also had significantly higher intakes of fat and $\mathrm{Na}$, and significantly lower intakes of protein and total carbohydrate (Table $1 ; P<0.001$ for all variables). Similarly, animals fed the cafeteria diet during pregnancy (CON-CAF and CAF-CAF) consumed more energy, fat and salt, and less protein and total carbohydrate than animals fed the control diet during pregnancy (CON-CON and CAF-CON), irrespective of their prepregnancy diet (Fig. 1, Table 1; $P<0 \cdot 001$ ). These effects remained when body weight was entered into analyses as a covariate. The effects of cafeteria feeding in the prepregnancy and pregnancy periods are also reflected in the data showing nutrient intakes as a percentage of total energy intakes (Fig. 2). Cafeteria-fed animals consumed a significantly greater percentage of their daily energy intake as fat, and significantly less as protein and carbohydrate $(P<0.001)$.

There was also an effect of pre-pregnancy diet on nutrient intakes during pregnancy, but only when body weight was entered as a covariate. Animals that had been fed the cafeteria diet in the pre-pregnancy period exhibited lower food intakes relative to body weight during pregnancy than those who had consumed the control diet pre-pregnancy. This was reflected in significantly lower intakes of all nutrients relative to body weight in the CAF-CON and CAF-CAF groups in comparison with the CON-CON and CON-CAF groups (Table 1; $P<0 \cdot 001$ ), with the exception of fat which did not reach statistical significance.

\section{Maternal body weight and composition}

All rats gained weight during the pre-pregnancy and pregnancy periods (Fig. 3). Rats fed the cafeteria diet during the pre-gestational period had a significantly increased weight gain before mating in comparison with those fed the control diet (CON, 116 (SEM 24) g; CAF, 142 (SEM 30) g; $P<0.001)$. Similarly, rats fed the cafeteria diet during pregnancy had a significantly increased gestational weight gain in comparison with those fed the control diet (CON, 90 (SEM 27) g, CAF, 115 (SEM 33) g; $P<0.001$ ), irrespective of the pre-gestational diet. Rats that remained on the cafeteria diet through the pre-gestation period and pregnancy (CAF$\mathrm{CAF}$ ) were therefore heavier than all the other groups throughout pregnancy. Weight gain slowed in rats switched to the control diet during pregnancy (CAF-CON), and these animals exhibited the lowest gestational weight gain overall.

At day 5 of gestation, animals that had been fed the cafeteria diet during the pre-gestation period had significantly heavier abdominal fat pads (Table 2). Gonadal fat pads were 75 and $73 \%$ heavier and perirenal fat pads 64 and $124 \%$ heavier in the CAF-CON and CAF-CAF groups, respectively, compared with the CON-CON group. Cafeteria-fed rats that were then transferred to the control diet during gestation (CAF-CON) exhibited a slowing of net fat deposition at the peri-renal site and gonadal sites in comparison with those that remained on the cafeteria diet during pregnancy (CAF-CAF). However, they still had significantly heavier fat depots at day 20 of gestation than those fed the control diet throughout the study (CON-CON). The significant effects of the pre-gestational cafeteria diet therefore persisted to day 20 of gestation, irrespective of gestational diet. Cafeteria feeding in the gestational period also increased the weight of these fat depots in comparison with those fed the control diet during gestation. Thus the heaviest average fat depot weights were observed in the CAF-CAF group.

Shifts in the weight of abdominal fat depots reflected changes in overall carcass fat (Fig. 4). At day 5 of gestation, animals that had been fed the cafeteria diet in the pre-gestational period had significantly higher body fat and lower $\mathrm{N}$ and water as a percentage of total body weight in comparison with animals fed the control diet in the pre-gestational period (Fig. 4(a); body fat - CON: 36\%, CAF: $43 \%$; $P<0.05$ ). Similarly, at day 20 of gestation, animals fed the cafeteria diet during gestation exhibited significantly higher body fat

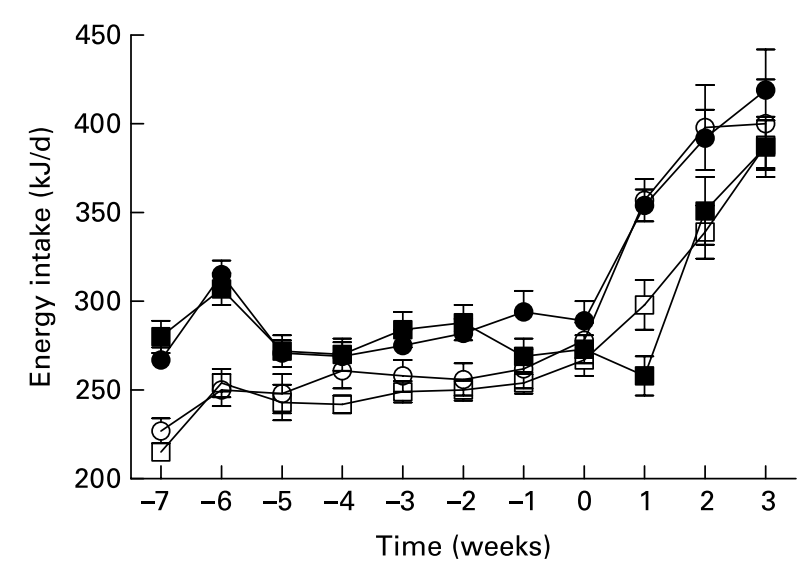

Fig. 1. Average daily energy intakes during each week of the pre-gestation and gestation periods. Values are means, with standard errors represented by vertical bars. Pre-gestational intakes (weeks -7 to 0 ) are shown for animals fed a control (CON-CON, $n 12(\square)$ and CON-CAF, $n 11(O)$ ) or cafeteria diet (CAF-CON, $n 11(\square)$ and CAF-CAF, $n 12(\bullet))$ before mating (pre-gestational CON v. CAF, $P<0.05$ ). Gestational intakes (weeks 1 to 3 ) are shown for animals fed a control (CON-CON, $n 6$ (weeks 2 and 3 ) to $n 12$ (week 1), or CAF-CON, $n 6$ (weeks 2 and 3) to $n 11$ (week 1)) or a cafeteria (CON-CAF, $n 6$ (weeks 2 and 3) to $n 11$ (week 1), or CAF-CAF, $n 6$ (weeks 2 and 3 ) to $n 12$ (week 1)) diet during pregnancy (gestational CON $v$. CAF, $P<0.05)$. 
Table 1. Average daily maternal intakes of energy and nutrients during the pre-gestational (weeks -7 to -1 ) and gestational (weeks 1 to 3 ) periods (Mean values with their standard errors)

\begin{tabular}{|c|c|c|c|c|c|c|c|c|c|c|c|c|}
\hline & \multicolumn{4}{|c|}{ Pre-gestational intakes } & \multicolumn{8}{|c|}{ Gestational intakes } \\
\hline & \multicolumn{2}{|c|}{ CON (n 23) } & \multicolumn{2}{|c|}{ CAF $(n 23)$} & \multicolumn{2}{|c|}{ CON-CON (n 12) } & \multicolumn{2}{|c|}{ CON-CAF $(n 11)$} & \multicolumn{2}{|c|}{ CAF-CON ( $n$ 11) } & \multicolumn{2}{|c|}{ CAF-CAF $(n 12)$} \\
\hline & Mean & SEM & Mean & SEM & Mean & SEM & Mean & SEM & Mean & SEM & Mean & SEM \\
\hline Energy intake $(\mathrm{MJ} / \mathrm{d})$ & 0.25 & 0.00 & $0 \cdot 28^{*}$ & 0.01 & 0.32 & 0.01 & $0.37 \dagger$ & 0.01 & $0.29 \ddagger$ & 0.02 & $0.37 \dagger \ddagger$ & 0.01 \\
\hline Fat intake (g/d) & 0.45 & 0.01 & $3.09^{*}$ & 0.07 & 0.58 & 0.03 & $4.23 \dagger$ & $0 \cdot 17$ & 0.53 & 0.03 & $4.45 \dagger$ & 0.21 \\
\hline Protein intake (g/d) & $2 \cdot 78$ & 0.05 & $2 \cdot 22^{*}$ & 0.05 & 3.81 & 0.19 & $2 \cdot 81 \dagger$ & 0.20 & $3.41 \ddagger$ & 0.23 & $2 \cdot 63 \dagger \ddagger$ & 0.13 \\
\hline Carbohydrate intake $(\mathrm{g} / \mathrm{d})$ & $11 \cdot 10$ & 0.22 & $7 \cdot 56^{\star}$ & 0.22 & 14.00 & 0.63 & $9.55 \dagger$ & 0.67 & $12 \cdot 58 \ddagger$ & 0.85 & $9 \cdot 16 \dagger \ddagger$ & 0.41 \\
\hline Na intake $(g / d)$ & 0.023 & 0.001 & $0.062^{*}$ & 0.002 & 0.0314 & 0.004 & $0.0842 \dagger$ & 0.003 & $0.0279 \ddagger$ & 0.002 & $0.0789 \dagger \ddagger$ & 0.004 \\
\hline
\end{tabular}

CON, control chow diet; CAF, cafeteria diet; CON-CON, control chow diet pre-gestation and during pregnancy; CON-CAF, control chow diet pre-gestation and cafeteria diet during pregnancy; CAF-CON, cafeteria diet pre-gestation and control chow diet during pregnancy; CAF-CAF, cafeteria diet pre-gestation and during pregnancy.

* Mean value was significantly different from that of the CON group $(P<0.001$, with or without body weight as a covariate).

$\dagger$ Mean value was significantly different from that of pregnancy CON-fed animals of the same pre-gestational diet group $(C O N-C O N$ or $C A F-C O N$ group) $(P<0.01$, with or without body weight as a covariate).

$\ddagger$ Mean value was significantly different from that of pre-gestation CON-fed animals of the same pregnancy diet group (CON-CON or CON-CAF group) $(P<0.001$, with body weight as a covariate).

and lower $\mathrm{N}$ and water as a percentage of total body weight in comparison with animals fed the control diet during gestation (Fig. 4(b); $P<0.05$ ). Mirroring observations of the weights of abdominal fat depots, the effects noted at day 20 of gestation did interact with the effects of the pre-gestational dietary treatment. Rats fed the cafeteria diet in the pre-gestational period and then transferred to the control diet during gestation (CAF-CON) exhibited similar total body fat at days 5 and 20 of gestation (41 and $42 \%$, respectively), whereas the CAF-CAF animals continued to deposit fat during gestation ( $45 \%$ at day 5 and $53 \%$ at day 20). Rats fed the control diet in the pre-gestational period and then transferred to the cafeteria diet during gestation (CON-CAF) exhibited an increase in body fat during pregnancy (body fat $38 \%$ at day 5 of gestation and $46 \%$ at day 20 of gestation). Although the increase was of similar magnitude to CAF-CAF animals, the degree of adiposity attained was

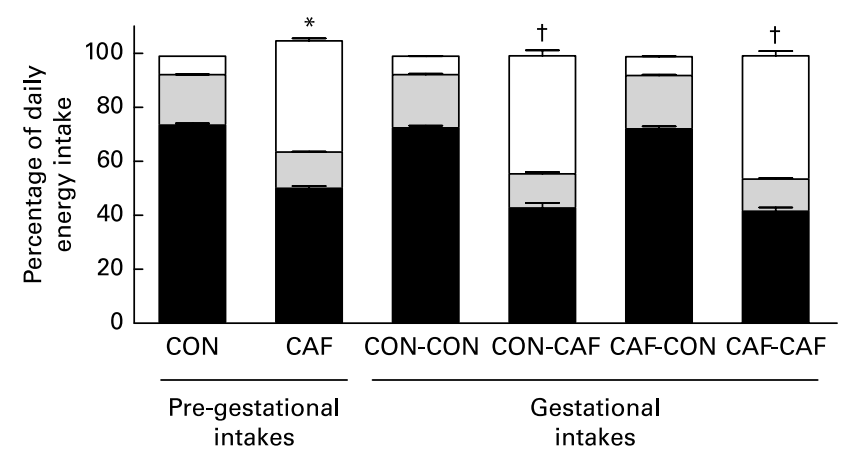

Fig. 2. Maternal nutrient intakes as a percentage of energy intake. Values are means, with standard errors represented by vertical bars. Pre-gestational intakes are shown for animals fed a control (CON, $n$ 23) or cafeteria (CAF $n$ 23) diet before mating. Gestational intakes are shown for animals fed a control (CON-CON, $n 6$ (weeks 2 and 3) to $n 12$ (week 1), or CAF-CON, $n 6$ (weeks 2 and 3 ) to $n 11$ (week 1)) or a cafeteria (CON-CAF, $n 6$ (weeks 2 and 3) to $n 11$ (week 1), or CAF-CAF, $n 6$ (weeks 2 and 3) to $n 12$ (week 1)) diet during pregnancy. There was a significant effect of pre-gestational $\left({ }^{\star} P<0.001\right)$ and gestational $(\dagger P<0.001)$ cafeteria feeding on the intakes of protein $(\square)$, carbohydrate $(\square)$ and fat $(\square)$ (all three macronutrients) during each respective period. There was no effect of pre-gestational diet on gestational intakes as a percentage of energy intakes. lower in the CON-CAF group (CON-CAF $46 \%$, CAF-CAF $53 \%$, at day 20 of gestation).

\section{Plasma metabolites}

Although they were markedly obese, the cafeteria-fed rats showed little evidence of metabolic disturbances. Plasma glucose and TAG concentrations at day 5 or 20 of gestation were unaffected by cafeteria feeding at any stage of the experiment (Table 3). TAG and total cholesterol concentrations increased between day 5 and day 20 of gestation in all groups of animals $(P<0.001)$. There was a tendency towards an interaction between the pre-gestational and gestational diets in their effects on plasma glucose concentrations at day 20 of gestation $(P<0.06)$, with the CON-CON rats exhibiting lower glucose concentrations than the three cafeteria-fed groups.

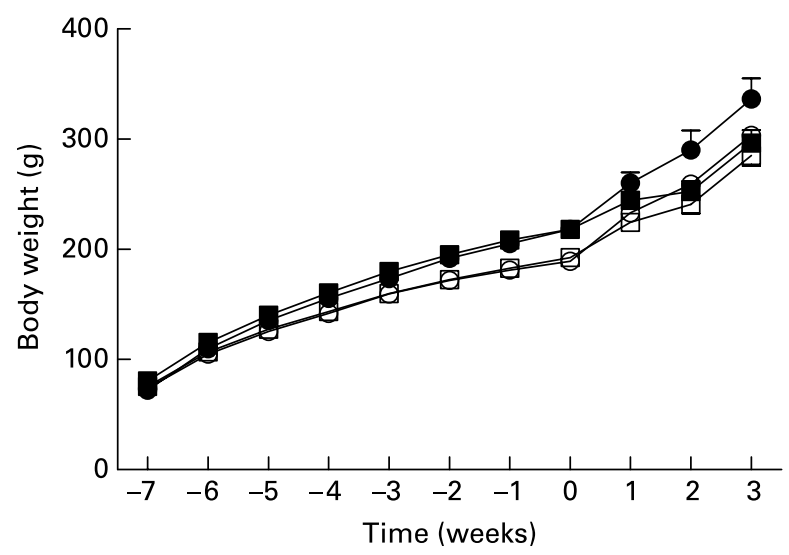

Fig. 3. Body-weight changes before and during pregnancy in rats fed a control (CON) or cafeteria (CAF) diet. Values are means, with standard errors represented by vertical bars, for $n 11$ or 12 (before day 5 of gestation) or $n 6$ (after day 5 of gestation). ( $\square$ ), CON-CON, control diet pre- and during pregnancy; $(O)$, CON-CAF, control diet pre-gestation, cafeteria diet during pregnancy; $(\mathbf{\square})$, CAF-CON, cafeteria diet pre-gestation, control diet during pregnancy; $(\bullet)$, CAF-CAF, cafeteria diet pre- and during pregnancy. Weight gain during the pre-gestational period was significantly higher in the CAF-fed animals $(P<0.001)$. Weight gain during the gestational period was significantly higher in animals fed a cafeteria diet during pregnancy, irrespective of pre-gestational diet $(P<0.001)$. 
Table 2. Maternal fat depot mass $\ddagger$

(Mean values with their standard errors for five or six observations per group)

\begin{tabular}{|c|c|c|c|c|c|c|c|c|}
\hline \multirow[b]{4}{*}{ Maternal diet } & \multicolumn{8}{|c|}{ Fat depot weight $(\mathrm{g})$} \\
\hline & \multicolumn{4}{|c|}{ Gonadal fat } & \multicolumn{4}{|c|}{ Peri-renal fat } \\
\hline & \multicolumn{2}{|c|}{ Day 5} & \multicolumn{2}{|c|}{ Day 20} & \multicolumn{2}{|c|}{ Day 5} & \multicolumn{2}{|c|}{ Day 20} \\
\hline & Mean & $\overline{\text { SEM }}$ & Mean & $\overline{\mathrm{SEM}}$ & Mean & $\overline{\text { SEM }}$ & Mean & SEM \\
\hline CON-CON & 4.22 & 1.34 & $7 \cdot 25$ & $1 \cdot 11$ & 3.07 & 0.99 & $6 \cdot 66$ & 1.47 \\
\hline CON-CAF & 4.81 & 1.40 & $8.00 \dagger$ & 3.67 & 4.41 & 1.34 & $7.97 \dagger$ & $2 \cdot 16$ \\
\hline CAF-CON & $8 \cdot 27^{\star}$ & 3.38 & $8 \cdot 29^{*}$ & 4.46 & $5 \cdot 62^{*}$ & 2.14 & $7.53^{*}$ & 2.00 \\
\hline CAF-CAF & $8.03^{\star}$ & 3.09 & $12 \cdot 34^{\star} \dagger$ & 4.44 & $7 \cdot 90^{\star}$ & 4.52 & $13 \cdot 80^{*} \dagger$ & 4.87 \\
\hline
\end{tabular}

CON-CON, control chow diet pre-gestation and during pregnancy; CON-CAF, control chow diet pre-gestation and cafeteria diet during pregnancy; CAF-CON, cafeteria diet pre-gestation and control chow diet during pregnancy; CAF-CAF, cafeteria diet pre-gestation and during pregnancy.

${ }^{*}$ Mean value was significantly different from that of pre-gestation CON-fed animals of the same pregnancy diet group $(P<0.05)$.

$\dagger$ Mean value was significantly different from that of pregnancy $\mathrm{CON}$-fed animals of the same pre-gestational diet group $(P<0.05)$.

$\ddagger$ Body weight was entered as a covariate for all analyses.

\section{Reproductive outcome}

One of the aims of the present study was to assess whether maternal obesity and/or the cafeteria diet had any impact upon the reproductive success of the rats, or their adaptation to pregnancy. All animals became pregnant and only one rat failed to carry pregnancy to day 20 of gestation. There was no effect of cafeteria feeding in either the pre-gestational period or during gestation on overall litter size (CON-CON, 12.3 (SEM 0.5); CON-CAF, 11.7 (SEM 0.5); CAF-CON, 13.0 (SEM 0.45); CAF-CAF, 11.3 (SEM 1.3)). Maternal plasma volume was determined as a measure of the reno-cardiovascular adaptation to pregnancy (Fig. 5). It was apparent that there was a significant increase in plasma volume between day 5 and day 20 of gestation in all groups $(P<0 \cdot 001)$. There was no evidence that either maternal obesity associated with cafeteria feeding in the pre-gestational period, or the cafeteria diet itself, had any impact upon this physiological adaptation to pregnancy.

\section{Fetal and placental growth}

Cafeteria feeding in pregnancy had no significant impact upon fetal growth, with fetal weight at day 20 in the CON-CAF group similar to that in the CON-CON group. However, there was clearly an impact of cafeteria feeding to induce obesity in the pre-gestation period, as shown by the significant effect of pre-gestational diet on fetal weight at day 20 $(P<0.001)$ (Fig. 6(a)). Fetuses of the CAF-CON and CAF-CAF groups were significantly smaller than those of the CON-CON and CON-CAF groups. In contrast to fetal weight, placental weight appeared to be sensitive only to the diet in pregnancy, with cafeteria feeding in pregnancy resulting in reduced placental size $(P<0.001)$ (Fig. 6(b)). The significant effects of pre-gestational diet on fetal weight and gestational diet on placental weight were reflected in the fetal: placental weight ratios (Fig. 6(c)). Whilst the cafeteria diet during the pre-gestational period significantly reduced the fetal:placental weight ratio $(P<0.001)$, the cafeteria diet during gestation significantly increased the fetal:placental
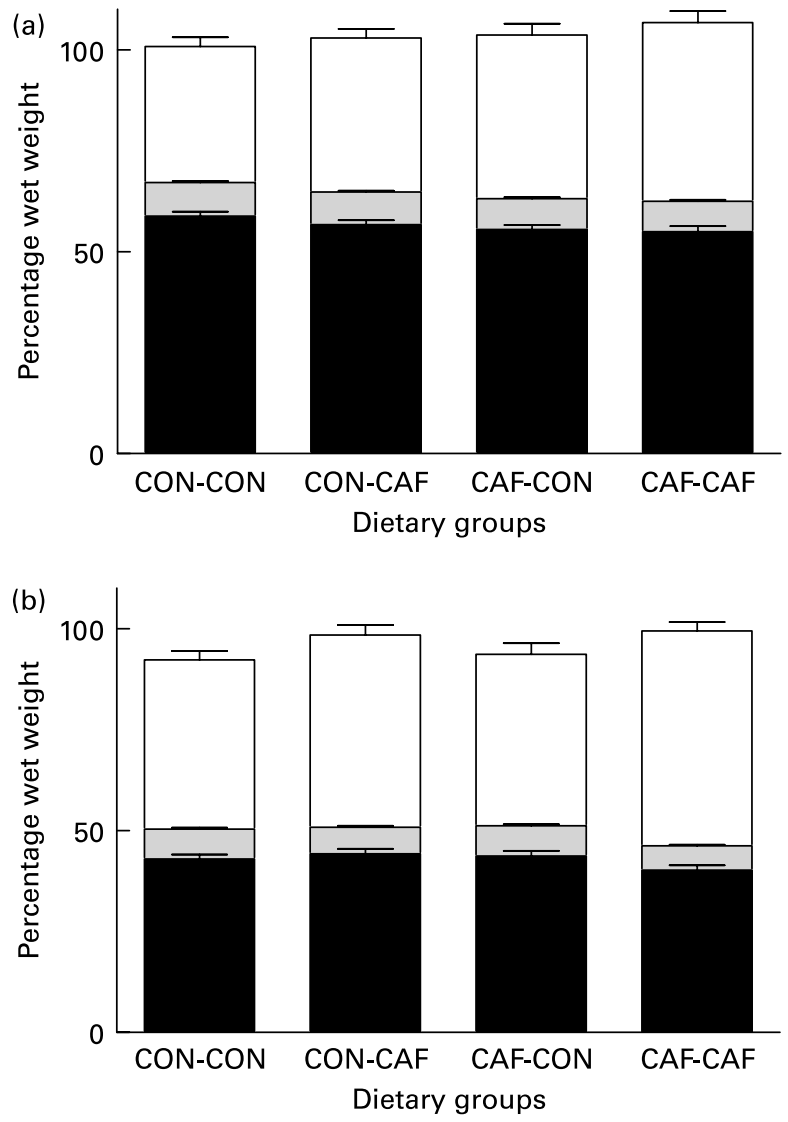

Fig. 4. Maternal body composition on days 5 (a) and 20 (b) of gestation. Values are means ( $n 5$ or 6 ), with standard errors represented by vertical bars. CON-CON, control diet pre- and during pregnancy; CON-CAF, control diet pre-gestation, cafeteria diet during pregnancy; CAF-CON, cafeteria diet pre-gestation, control diet during pregnancy; CAF-CAF, cafeteria diet pre- and during pregnancy. There was a significant effect of pre-gestational diet on carcass water $(\square), N(\square)$ and fat $(\square)$ content at day 5 of gestation $(P<0.05)$. There was a similar significant effect of gestational diet on carcass water, $\mathrm{N}$ and fat at day 20 of gestation and this interacted with the effects of pre-gestational diet $(P<0.05)$. 
Table 3. Concentrations of glucose, cholesterol and TAG in maternal plasma (Mean values with their standard errors for five or six observations per group)

\begin{tabular}{|c|c|c|c|c|c|c|c|c|c|c|c|c|}
\hline \multirow[b]{3}{*}{ Maternal diet } & \multicolumn{4}{|c|}{ Glucose $(\mathrm{mmol} / \mathrm{l})$} & \multicolumn{4}{|c|}{ Cholesterol (mmol/l) } & \multicolumn{4}{|c|}{$\mathrm{TAG}(\mathrm{mmol} / \mathrm{l})$} \\
\hline & \multicolumn{2}{|c|}{ Day 5} & \multicolumn{2}{|c|}{ Day 20} & \multicolumn{2}{|c|}{ Day 5} & \multicolumn{2}{|c|}{ Day 20} & \multicolumn{2}{|c|}{ Day 5} & \multicolumn{2}{|c|}{ Day 20} \\
\hline & Mean & SEM & Mean & SEM & Mean & SEM & Mean & SEM & Mean & SEM & Mean & SEM \\
\hline CON-CON & 3.49 & 0.43 & $2.09 \dagger$ & 0.13 & 1.54 & 0.11 & $2 \cdot 21^{\star \star \star}$ & 0.23 & 0.85 & 0.18 & $2 \cdot 05^{\star}$ & 0.49 \\
\hline CON-CAF & $2 \cdot 75$ & 0.23 & $3 \cdot 11$ & 0.22 & $1 \cdot 11$ & 0.21 & $1.77^{\star \star \star}$ & 0.19 & 0.43 & 0.19 & $1.48^{*}$ & 0.46 \\
\hline CAF-CON & 3.54 & 0.27 & 2.96 & 0.42 & 1.55 & 0.11 & $1.88^{* \star *}$ & 0.30 & 0.65 & 0.15 & $2.99^{\star}$ & 0.50 \\
\hline CAF-CAF & 3.72 & 0.85 & 2.91 & 0.26 & 1.60 & 0.10 & $2 \cdot 08^{\star \star \star}$ & 0.14 & 0.52 & $0 \cdot 16$ & $2 \cdot 20^{\star}$ & 0.63 \\
\hline
\end{tabular}

CON-CON, control chow diet pre-gestation and during pregnancy; CON-CAF, control chow diet pre-gestation and cafeteria diet during pregnancy; CAF-CON, cafeteria diet pre-gestation and control chow diet during pregnancy; CAF-CAF, cafeteria diet pre-gestation and during pregnancy.

Mean value was significantly different from that on day $5:{ }^{*} P<0.05,{ }^{\star \star \star} P<0.001$.

$\dagger$ Mean value was marginally different from those of the three cafeteria-fed groups on day $20(P<0.06)$.

weight ratio $(P<0 \cdot 001)$. The lowest fetal:placental weight ratio was therefore observed in the CAF-CON group, and the highest in the CON-CAF group. Although the CAF-CAF and CON-CON groups exhibited similar fetal:placental weight ratios, the combined weight of the fetus and placenta was lower in the CAF-CAF group. Although cafeteria feeding to induce maternal obesity in the pre-gestational period was associated with fetal growth restriction, most fetal organs remained in proportion to body weight. This is reflected in the lack of significant differences observed when fetal organ weights were analysed with body weight as a covariate (Table 4). The exceptions to this were the kidneys, where there was some evidence that cafeteria diet consumption in pregnancy reduced renal mass $(P<0.05)$, and the brain, where a significant interaction between pre-gestational and gestational diets $(P<0.01)$ indicated that the effect of the cafeteria diet during pregnancy on brain weight differed according to pre-gestational diet.

\section{Discussion}

In contrast to the more immediate consequences for maternal and neonatal health, the effects of maternal obesity on the long-term health of the offspring remain poorly understood. Animal studies support the hypothesis that there may be long-term implications for offspring metabolic function and future obesity risk ${ }^{(20-24)}$. However, it is not clear whether such effects are the result of maternal obesity per se or the diets fed to induce obesity. The primary aim of the present investigation was to develop a robust model for the evaluation of the independent effects of a cafeteria diet and the intra-uterine environment associated with maternal obesity upon the outcome of pregnancy. The feeding regimen proved effective in inducing obesity without impacting on reproductive success. The study successfully demonstrated that there were differential effects of maternal body fatness and maternal diet upon fetal growth and that the model will therefore be a suitable vehicle for future investigation of the programming of disease risk in older offspring subjected to these protocols.

Cafeteria feeding provides a useful alternative to the feeding of purified high-fat diets to induce obesity, and induces persistent hyperphagia and increased energy intakes ${ }^{(28,29)}$ as a result of the variety and novelty of the foods available. It is noteworthy that in our hands the increase in energy intake was lower than in other reports using the cafeteria protocol. Other studies have noted that daily energy intakes of cafeteria-fed rats are between 160 and $250 \mathrm{~kJ} / \mathrm{d}$ higher than those of control animals ${ }^{(22,29-31)}$. On average, daily energy intake was $30 \mathrm{~kJ} / \mathrm{d}$ greater in CAF-fed compared with chow-fed rats, although in the first 2 weeks of the protocol rats consumed approximately $60 \mathrm{~kJ} / \mathrm{d}$ more. Variability in
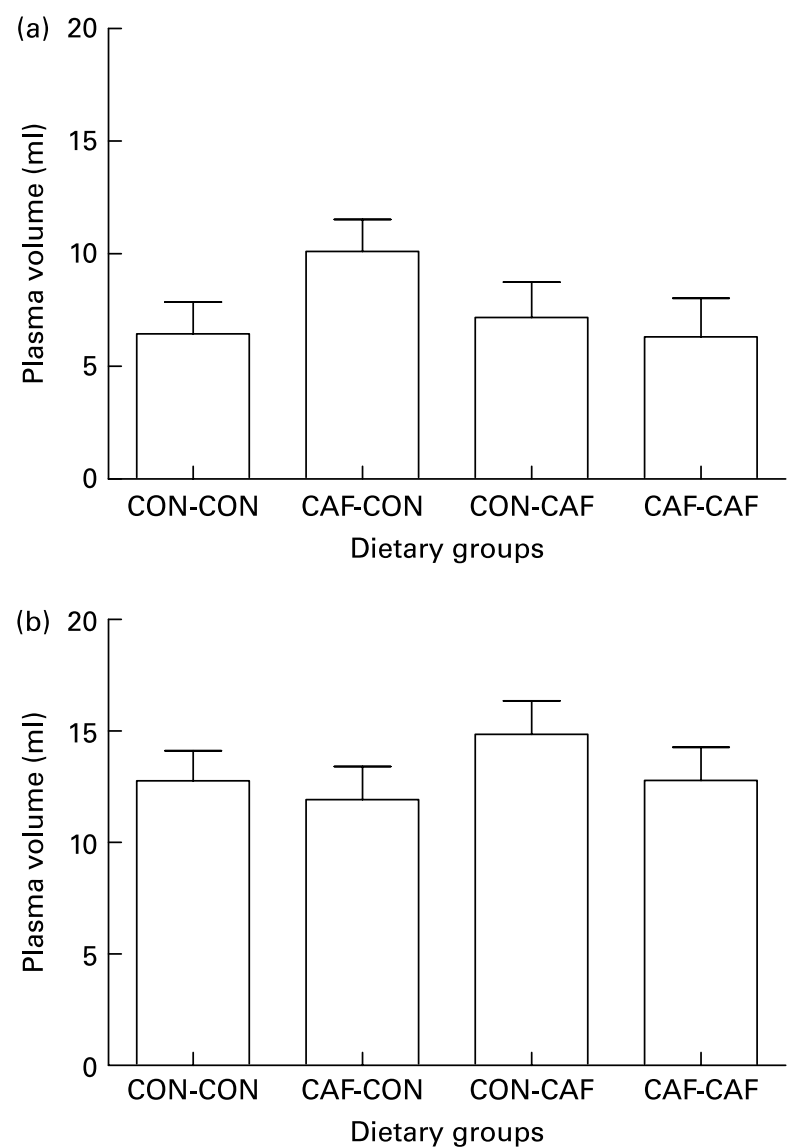

Fig. 5. Maternal plasma volume on days 5 (a) and 20 (b) of gestation. Values are means ( $n$ 6), with standard errors represented by vertical bars. CON-CON, control diet pre- and during pregnancy; CAF-CON, cafeteria diet pre-gestation, control diet during pregnancy; CON-CAF, control diet pre-gestation, cafeteria diet during pregnancy; CAF-CAF, cafeteria diet preand during pregnancy. Plasma volume was influenced by the gestational stage $(P<0.001)$. 

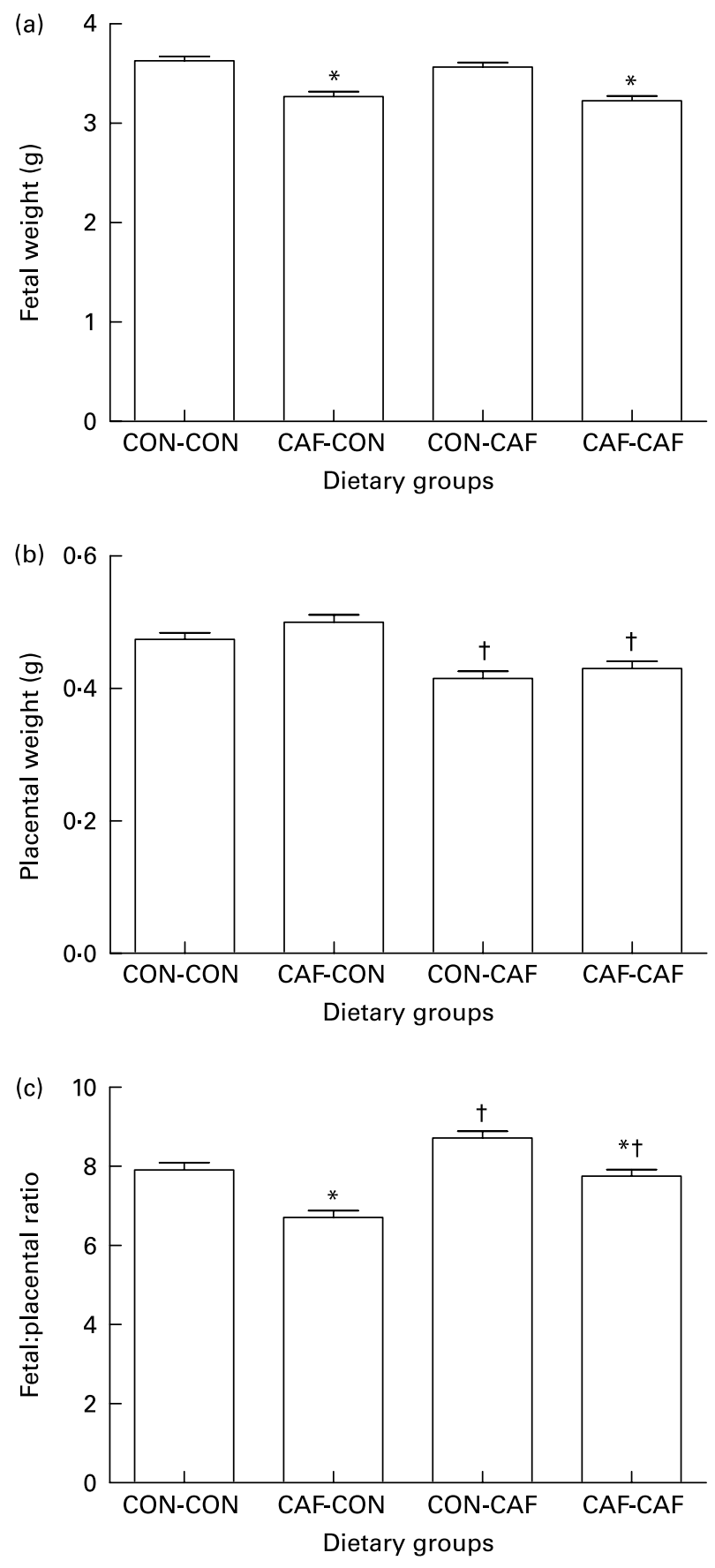

Fig. 6. Fetal (a) and placental (b) weights and their ratio (c) in pregnant rats on day 20 of gestation fed the control (CON) or cafeteria (CAF) diet. Values are means ( $n$ 59-73), with standard errors represented by vertical bars. CON-CON, control diet pre- and during pregnancy; CAF-CON, cafeteria diet pre-gestation, control diet during pregnancy; CON-CAF, control diet pre-gestation, cafeteria diet during pregnancy; CAF-CAF, cafeteria diet preand during pregnancy. (a) Fetal weight on day 20 of gestation was influenced by the pre-gestational diet $(P<0.001)$. ${ }^{*}$ Mean value was significantly different from that of pre-gestation $\mathrm{CON}$-fed animals of the same pregnancy diet group $(P<0.001)$. (b) Placental weight on day 20 of gestation was influenced by the gestational diet $(P<0.001)$. † Mean value was significantly different from that of pregnancy CON-fed animals of the same pre-gestational diet group $(P<0.001)$. (c) Fetal:placental ratio on day 20 of gestation was influenced by the pre-gestational diet $(P<0.001)$ and gestational diet $(P<0.001)$. * Mean value was significantly different from that of pre-gestation CON-fed animals of the same pregnancy diet group $(P<0.001)$. † Mean value was significantly different from that of pregnancy $\mathrm{CON}$-fed animals of the same pre-gestational diet group $(P<0.001)$. impact of CAF feeding may be explained by differences in age and strain of the rats under study ${ }^{(32)}$, but it seems most likely that the profile of foods used explains the present results. Whilst we used a panel of twelve food items, Shafat et al. ${ }^{(29)}$, for example, used thirty-six, thereby maintaining novelty for longer. This does not impact strongly on our conclusions as the aim of the study, that is the induction of maternal obesity, was achieved even with less marked hyperphagia.

The increased energy intakes of the cafeteria-fed rats in the present study were associated with a significant change in the composition of their diets and thus the proportion of energy consumed as fat, carbohydrate or protein. Their substantially higher fat intake (CON-CON: 2.44 (SEM 0.12) g/d per $\mathrm{kg}$ body weight $v$. CAF-CAF: $16 \cdot 28$ (SEM 0.80) g/d per kg body weight) meant that cafeteria animals were consuming a much greater proportion of their daily energy intake from fat and less from protein and carbohydrate, similar to other cafeteria studies ${ }^{(22,29,33,34)}$. CAF-fed animals also had higher intakes of $\mathrm{Na}$. The use of dietary patterns as a measure of exposure in human studies of diet-disease relationships provides a useful alternative to the assessment of single nutrients or foods. A recent review of such studies demonstrated an inverse association between 'prudent' dietary patterns (i.e. in line with UK Department of Health recommendations) and all-cause mortality and CVD risk ${ }^{(35)}$. The dietary pattern observed with a cafeteria feeding system reflects that of the 'non-prudent' human ${ }^{(35,36)}$, and avoids the very high intakes of specific and potentially biologically active fatty acids, which are observed in other models of high-fat feeding. It is therefore considered an effective tool for modelling the effects of 'non-prudent' dietary patterns in humans, rather than for investigating the impact of controlled alterations in the intake of specific nutrients.

The dietary pattern of pregnant rats fed a cafeteria diet would be expected to have permanent effects on the metabolic function and health of the offspring in later life, as previous studies have shown such effects in response to altering the intakes of individual nutrients during pregnancy. For example, feeding a low-protein or high-fat diet to rats during pregnancy has been shown to alter renal and cardiovascular function ${ }^{(37-40)}$, insulin sensitivity $^{(41,42)}$ and dietary behaviours ${ }^{(22,43)}$ in postnatal life. Although the CAF-fed rats had lower intakes of protein, they were not consuming a low-protein diet and their intakes were above the recommendations for growing and pregnant animals $^{(44)}$. The present study aimed to use a cross-over experimental design to develop a model which distinguishes between the effects of dietary treatment and obesity per se.

Cafeteria feeding in the pre-gestational period was effective in inducing obesity, as shown in other studies ${ }^{(28,29)}$. This is demonstrated by the significantly heavier abdominal fat depots and higher percentage total body fat in cafeteria-fed rats in comparison with controls at day 5 of gestation. Rats that were then transferred to the control diet at mating (CAF-CON) remained significantly fatter throughout pregnancy when compared with those who had consumed the control diet throughout the study (CON-CON). We propose that this group provides an opportunity to examine the effects of maternal obesity per se, without the confounding effects of an altered maternal diet during pregnancy. Similarly, rats fed a pre-gestational control diet and then transferred to the 
Table 4. Fetal organ size relative to body weight on day 20 of gestation (Mean values with their standard errors for 59-73 observations per group)

\begin{tabular}{|c|c|c|c|c|c|c|c|c|c|c|}
\hline \multirow[b]{3}{*}{ Maternal diet } & \multicolumn{10}{|c|}{ Organ weight/body weight (\%) } \\
\hline & \multicolumn{2}{|c|}{ Liver } & \multicolumn{2}{|c|}{ Brain $^{\star *}$} & \multicolumn{2}{|c|}{ Heart } & \multicolumn{2}{|c|}{ Left kidney } & \multicolumn{2}{|c|}{ Right kidney } \\
\hline & Mean & SEM & Mean & SEM & Mean & SEM & Mean & SEM & Mean & SEM \\
\hline CON-CON & 0.261 & 0.005 & 0.133 & 0.002 & 0.019 & 0.001 & 0.018 & 0.002 & 0.014 & 0.001 \\
\hline CON-CAF & 0.247 & 0.006 & 0.126 & 0.004 & 0.018 & 0.001 & $0.013 \dagger$ & 0.001 & 0.014 & 0.001 \\
\hline CAF-CON & 0.225 & 0.007 & 0.124 & 0.003 & 0.019 & 0.001 & 0.014 & 0.001 & 0.014 & 0.001 \\
\hline CAF-CAF & 0.220 & 0.005 & 0.136 & 0.002 & 0.017 & 0.001 & $0.012 \dagger$ & 0.001 & 0.011 & 0.000 \\
\hline
\end{tabular}

CON-CON, control chow diet pre-gestation and during pregnancy; CON-CAF, control chow diet pre-gestation and cafeteria diet during pregnancy; CAF-CON, cafeteria diet pre-gestation and control chow diet during pregnancy; CAF-CAF, cafeteria diet pre-gestation and during pregnancy.

† Mean value was significantly different from that of pregnancy CON-fed animals of the same pre-gestational diet group $(P<0.05)$

${ }^{\star}$ There was an interaction between pre-gestational diet and gestational diet in their effects on the weight of the brain $(P<0.01)$

cafeteria diet at mating (CON-CAF) provide an opportunity to investigate the effects of cafeteria feeding per se. Although these animals did exhibit increased body fatness in comparison with controls by day 20 of gestation inevitably, this was not to the extent observed in rats that had been fed the cafeteria diet in the pre-gestational period to induce obesity at mating.

Having demonstrated the effectiveness of the cafeteria feeding system in inducing obesity before mating and the suitability of the cross-over experimental design, it was important to investigate whether the dietary treatments had impacted upon reproductive outcome. No differences were apparent in breeding success or litter size between the control and cafeteria-fed rats, supporting the suitability of this dietary regimen for studies in pregnancy. There was also no effect on plasma volume expansion during pregnancy, indicating that the maternal reno-vascular adaptation to pregnancy occurred as normal ${ }^{(45)}$.

In the present study, cafeteria feeding had little impact upon circulating maternal plasma glucose, TAG or cholesterol. Average plasma glucose concentrations were lower in animals fed the control diet at day 20 of gestation in comparison with animals fed the cafeteria diet in the pre-gestational and/or gestational periods, but this did not reach statistical significance $(P=0 \cdot 06)$. Although high-fat feeding over a prolonged period of time in rodents generally leads to moderate hyperglycaemia and hypertriacylglycerolaemia, the literature demonstrates high variability in the effects of high-fat feeding on circulating markers of glucose and lipid metabolism ${ }^{(46)}$. This may be explained by the different fatty acid composition and total fat content of the high-fat diets used, and the length of time that the animals consumed the diets. In addition, circulating metabolites may have been affected by the type of profile of cafeteria foods offered on the day of blood sampling and the timing of blood sampling in the postprandial period. In agreement with our data, most studies show rats to be resistant to the development of hypercholesterolaemia unless fed a high-fat high-cholesterol $\operatorname{diet}^{(46,47)}$. Whilst the present study demonstrates no gross disturbance of glucose and lipid homeostasis, it must be noted that blood was collected from animals in the fed state and that the metabolic adjustments acting to maintain maternal circulating glucose and cholesterol levels may themselves impact upon the intra-uterine environment.
The importance of distinguishing between the effects of dietary intervention and obesity per se is evidenced by their differing effects on fetal and placental weight. Interestingly, the pre-gestational cafeteria diet significantly reduced fetal weight at day 20 of gestation, irrespective of diet during pregnancy. Transferring to the control diet at mating did not, therefore, 'rescue' the fetus from the growth-restricting effects of maternal obesity in this model. Cafeteria feeding during gestation only did not impact on fetal weight, similarly to that observed by Bayol et al. ${ }^{(48)}$, supporting the notion that it is maternal obesity rather than the associated diet which is responsible for the fetal growth restriction. The growthrestricting effects of maternal obesity in this model do not appear to be mediated by a restriction of placental growth, as there was no effect of pre-gestational cafeteria feeding on placental weight. Instead, maternal obesity may be associated with altered placental nutrient transfer or utilisation in the feto-placental tissues ${ }^{(49,50)}$.

Within the animal literature, it is difficult to directly assess the impact of maternal obesity on birth weight, as in most studies the effects observed could be attributed to the high-fat feeding associated with it. High-fat feeding in the pre-gestational and gestational periods significantly increased neonatal weights in some studies (mouse ${ }^{(20,49)}$ ) but not others $\left(\mathrm{rat}^{(21)}\right.$ and mouse $\left.{ }^{(51,52)}\right)$. A study which used pre-gestational intragastric cannulation to isolate the effects of maternal obesity from those of the maternal diet showed no effect of obesity on birth weight ${ }^{(24)}$. Within the human literature, maternal obesity is primarily associated with fetal overgrowth $^{(4,53)}$, although a recent prospective study of pregnancy outcome in obese women found an increased risk of both small- and large-for-gestational-age infants ${ }^{(54)}$. It should also be noted that maternal obesity is a risk factor for preterm delivery, which is often associated with small-for-gestational-age fetuses. It is hypothesised that maternal obesity augments the increase in insulin resistance usually observed in pregnancy, increasing fuel supply to the fetus ${ }^{(53)}$. Differences observed in the effects of maternal obesity on fetal growth may therefore relate to the nature of the metabolic disturbance observed in each particular species and strain used, and differences in the diets associated with obesity in each animal model. Improved understanding of the interactions between maternal diet and adiposity in their effects on fetal development is required. 
In conclusion, the present study has developed a robust model for the evaluation of the independent effects of cafeteria feeding and maternal obesity. Cafeteria feeding from weaning and for a period of 8 weeks was effective in inducing obesity without impacting on reproductive success. The preliminary data have shown interesting effects of maternal obesity induced by cafeteria feeding in the pre-gestational period on fetal and placental growth, which differed from the effects of cafeteria feeding during the pregnancy period alone. Future work with this model will improve understanding of the complex interactions between dietary- and obesity-related factors during pregnancy in their effects on fetal development and postnatal metabolic function.

\section{Acknowledgements}

The technical assistance of C. Armett, S. Kirkland and R. Plant is gratefully acknowledged.

The present study was funded by Hacettepe University (Ankara, Turkey).

S. C. L.-E. and S. M. designed the experiment. A. A. performed the experimental and laboratory analyses, and collated the data. S. C. L.-E. and S. M. performed the statistical analyses. S. M., A. A. and S. C. L.-E. wrote the manuscript. All authors read and approved the findings.

There are no conflicts of interest.

\section{References}

1. British Heart Foundation (2008) European Cardiovascular Disease Statistics. London: British Heart Foundation.

2. Ogden CL, Carroll MD \& Flegal KM (2008) High body mass index for age among US children and adolescents, 2003-2006. JAMA 299, 2401-2405.

3. Foresight (2007) Tackling Obesities: Future Choices - Modelling Future Trends in Obesity \& Their Impact on Health. London: The Stationery Office.

4. Weiss JL, Malone FD, Emig D, et al. (2004) Obesity, obstetric complications and Cesarean delivery rate - a population-based screening study. Am J Obstet Gynecol 190, 1091-1097.

5. Walsh SW (2007) Obesity: a risk factor for preeclampsia. Trends Endocrinol Metab 18, 365-370.

6. Dixit A \& Girling JC (2008) Obesity and pregnancy. J Obstet Gynaecol 28, 14-23.

7. Chu SY, Kim SY, Schmid CH, et al. (2007) Maternal obesity and risk of Cesarean delivery: a meta-analysis. Obes Rev 8, 385-394.

8. Parsons TJ, Power C \& Manor O (2001) Fetal and early life growth and body mass index from birth to early adulthood in 1958 British cohort: longitudinal study. $\mathrm{Br}$ Med $J$ 323, $1331-1335$

9. Stettler N, Tershakovec AM, Zemel BS, et al. (2000) Early risk factors for increased adiposity: a cohort study of African American subjects followed from birth to young adulthood. Am J Clin Nutr 72, 378-383.

10. Whitaker RC (2004) Predicting preschooler obesity at birth: the role of maternal obesity in early pregnancy. Pediatrics 114, e29-e36.

11. Lawlor DA, Smith GD, O'Callaghan M, et al. (2007) Epidemiologic evidence for the fetal overnutrition hypothesis: findings from the Mater-University Study of Pregnancy and its outcomes. Am J Epidemiol 165, 418-424.

12. Smith GD, Steer C, Leary S, et al. (2007) Is there an intrauterine influence on obesity? Evidence from parent-child associations in the Avon Longitudinal Study of Parents and Children (ALSPAC). Arch Dis Child 92, 876-880.

13. Lake JK, Power C \& Cole TJ (1997) Child to adult body mass index in the 1958 British birth cohort: associations with parental obesity. Arch Dis Child 77, 376-381.

14. Curhan GC, Chertow GM, Willett WC, et al. (1996) Birth weight and adult hypertension and obesity in women. Circulation 94, 1310-1315.

15. Fall CHD, Osmond C, Barker DJP, et al. (1995) Fetal and infant growth and cardiovascular risk-factors in women. $\mathrm{Br}$ Med J 310, 428-432.

16. Pettitt DJ, Baird HR, Aleck KA, et al. (1983) Execessive obesity in offspring of Pima Indian women with diabetes during pregnancy. New Engl J Med 308, 242-245.

17. Gillman MW, Rifas-Shiman S, Berkey CS, et al. (2003) Maternal gestational diabetes, birth weight, and adolescent obesity. Pediatrics 111, e221-e226.

18. Silverman BL, Rizzo T, Green OC, et al. (1990) Long-term prospective evaluation of offspring of diabetic mothers. In $3 \mathrm{rd}$ International Workshop Conference on Gestational Diabetes Mellitus, 8-10 November 1990, Chicago, IL, pp. 121-125. Alexandria, VA: American Diabetes Association.

19. Langley-Evans SC (2006) Developmental programming of health and disease. Proceedings of the Nutrition Society 65, 97-105.

20. Samuelsson AM, Matthews PA, Argenton M, et al. (2008) Dietinduced obesity in female mice leads to offspring hyperphagia, adiposity, hypertension, and insulin resistance - a novel murine model of developmental programming. Hypertension 51, 383-392.

21. Chen H, Simar D, Lambert K, et al. (2008) Maternal and postnatal overnutrition differentially impact appetite regulators and fuel metabolism. Endocrinology 149, 5348-5356.

22. Bayol SA, Farrington SJ \& Stickland NC (2007) A maternal 'junk food' diet in pregnancy and lactation promotes an exacerbated taste for 'junk food' and a greater propensity for obesity in rat offspring. Br J Nutr 98, 843-851.

23. Bayol SA, Simbi BH, Bertrand JA, et al. (2008) Offspring from mothers fed a 'junk food' diet in pregnancy and lactation exhibit exacerbated adiposity that is more pronounced in females. J Physiol (Lond) 586, 3219-3230.

24. Shankar K, Harrell A, Liu XL, et al. (2008) Maternal obesity at conception programs obesity in the offspring. Am J Physiol Regul Integr Comp Physiol 294, R528-R538.

25. Blair ML \& Mickelsen D (2006) Plasma protein and blood volume restitution after hemorrhage in conscious pregnant and ovarian steroid-replaced rats. Am J Physiol Regul Integr Comp Physiol 290, R425-R434.

26. Langley SC \& York DA (1990) Increased type II glucocorticoid receptor numbers and glucocorticoid-sensitive enzyme activities in the brain of the obese Zucker rat. Brain Res 533, 268-274.

27. Trinder P (1969) Determination of blood glucose using a oxidase-peroxidase system with a non-carcinogenic chromogen. J Clin Pathol 22, 158-161.

28. Rothwell NJ \& Stock MJ (1979) Regulation of energy balance in 2 models of reversible obesity in the rat. J Comp Physiol Psychol 93, 1024-1034.

29. Shafat A, Murray B \& Rumsey D (2009) Energy density in cafeteria diet induced hyperphagia in the rat. Appetite 52, $34-38$.

30. Rozen R, Brigant L \& Apfelbaum M (1994) Effects of cycles of food restriction followed by ad libitum refeeding on body composition and energy expenditure in obese rats. Am J Clin Nutr 59, 560-565.

31. Rothwell NJ \& Stock MJ (1982) Effects of feeding a palatable cafeteria diet on energy balance in young and adult lean Zucker rats. Br J Nutr 47, 461-471. 
32. Rothwell NJ, Saville ME \& Stock MJ (1982) Effects of feeding a cafeteria diet on energy balance and diet-induced thermogenesis in 4 strains of rat. $J$ Nutr 112, 1515-1524.

33. Esteve M, Rafecas I, Fernández-López JA, et al. (1994) Effect of a cafeteria diet on energy intake and balance in Wistar rats. Physiol Behav 56, 65-71.

34. Llado I, Pico C, Palou A, et al. (1995) Protein and amino acid intake in cafeteria fed obese rats. Physiol Behav 58, 513-519.

35. Kant AK (2004) Dietary patterns and health outcomes. $J$ Am Diet Assoc 104, 615-635.

36. Crozier SR, Robinson SM, Borland SE, et al. (2006) Dietary patterns in the Southampton Women's Survey. Eur J Clin Nutr 60, 1391-1399.

37. Khan IY, Dekou V, Douglas G, et al. (2005) A high-fat diet during rat pregnancy or suckling induces cardiovascular dysfunction in adult offspring. Am J Physiol Regul Integr Comp Physiol 288, R127-R133.

38. Langley-Evans SC, Welham SJM \& Jackson AA (1999) Fetal exposure to a maternal low protein diet impairs nephrogenesis and promotes hypertension in the rat. Life Sci 64, 965-974.

39. Nwagwu MO, Cook A \& Langley-Evans SC (2000) Evidence of progressive deterioration of renal function in rats exposed to a maternal low-protein diet in utero. Br J Nutr 83, 79-85.

40. Bellinger L, Sculley DV \& Langley-Evans SC (2006) Exposure to undernutrition in fetal life determines fat distribution, locomotor activity and food intake in ageing rats. Int $J$ Obes 30, 729-738.

41. Buckley AJ, Keserü B, Briody J, et al. (2005) Altered body composition and metabolism in the male offspring of high fat-fed rats. Metabolism 54, 500-507.

42. Fernandez-Twinn DS, Wayman A, Ekizoglou S, et al. (2005) Maternal protein restriction leads to hyperinsulinemia and reduced insulin-signaling protein expression in 21-mo-old female rat offspring. Am J Physiol Regul Integr Comp Physiol 288, R368-R373.

43. Bellinger L, Lilley C \& Langley-Evans SC (2004) Prenatal exposure to a maternal low-protein diet programmes a preference for high-fat foods in the young adult rat. Br J Nutr 92, $513-520$.
44. Institute for Laboratory Animal Research (1995) Nutrient Requirements of Laboratory Animals, 4th ed. Washington, DC: National Academies Press.

45. Conrad KP (2004) Mechanisms of renal vasodilation and hyperfiltration during pregnancy. J Soc Gynecol Investig 11, $438-448$.

46. Buettner R, Scholmerich J \& Bollheimer LC (2007) High-fat diets: modeling the metabolic disorders of human obesity in rodents. Obesity 15, 798-808.

47. Chiang MT, Chen YC \& Huang AL (1998) Plasma lipoprotein cholesterol levels in rats fed a diet enriched in cholesterol and cholic acid. Int $J$ Vitam Nutr Res 68, 328-334

48. Bayol SA, Simbi BH \& Stickland NC (2005) A maternal cafeteria diet during gestation and lactation promotes adiposity and impairs skeletal muscle development and metabolism in rat offspring at weaning. $J$ Physiol (London) 567, 951-961.

49. Jones HN, Woollett LA, Barbour N, et al. (2008) High-fat diet before and during pregnancy causes marked up-regulation of placental nutrient transport and fetal overgrowth in C57/BL6 mice. In The 55th Annual Meeting of the Society for Gynecologic Investigation, 26-29 March 2008, San Diego, CA, pp. 271-278.

50. Osgerby JC, Gadd TS \& Wathes DC (2003) Effect of maternal body condition on placental and fetal growth and the insulinlike growth factor axis in Dorset ewes. Reproduction 125, 717-731.

51. Gupta A, Srinivasan M, Thamadilok S, et al. (2009) Hypothalamic alterations in fetuses of high fat diet-fed obese female rats. $J$ Endocrinol 200, 293-300.

52. Tozuka Y, Wada E \& Wada K (2009) Diet-induced obesity in female mice leads to peroxidized lipid accumulations and impairment of hippocampal neurogenesis during the early life of their offspring. FASEB $J \mathbf{2 3}, 1920-1934$.

53. King JC (2006) Maternal obesity, metabolism and pregnancy outcomes. Annu Rev Nutr 26, 271-291.

54. Rajasingam D, Seed PT, Briley AL, et al. (2009) A prospective study of pregnancy outcome and biomarkers of oxidative stress in nulliparous obese women. Am J Obstet Gynecol 200, 395.e1-395.e9. 